\title{
Substance $P$ enhances BMSC osteogenic differentiation via autophagic activation
}

\author{
WEN GENG $^{1,2 *}$, HUIMIN SHI ${ }^{3 *}$, XIMIN ZHANG $^{1}$, WEI TAN $^{1}$, YUAN CAO $^{1}$ and RONGCHENG MEI ${ }^{1}$ \\ ${ }^{1}$ Department of Orthopaedics, Xiangyang Central Hospital, Affiliated Hospital of Hubei University of Arts and Science, \\ Xiangyang, Hubei 441021; ${ }^{2}$ Department of Ophthalmology, Shengjing Hospital, China Medical University, Shenyang, \\ Liaoning 110004; ${ }^{3}$ Department of Ophthalmology, Xiangyang Central Hospital, Affiliated Hospital of \\ Hubei University of Arts and Science, Xiangyang, Hubei 441021, P.R. China
}

Received November 15, 2018; Accepted April 12, 2019

DOI: $10.3892 / \mathrm{mmr} .2019 .10257$

\begin{abstract}
Bone mesenchymal stem cells (BMSCs) are the most commonly investigated progenitor cells in bone tissue engineering for treating severe bone defects. Strategies for regulating $\mathrm{BMSC}$ differentiation fate have received wide attention, in which redox homeostasis plays an important role due to the change in energy metabolism during stem cell differentiation. In the present study, it was observed that autophagic activity was induced along with BMSC osteogenic differentiation and subsequently regulated reactive oxygen species (ROS) generation and the level of osteogenesis. Furthermore, it was also observed that neuropeptide substance $\mathrm{P}$ (SP) administration could enhance the autophagic activity in rat BMSCs via the AMPK and mTOR pathways, as well as decreasing ROS generation and promoting osteogenic differentiation. Inhibition of autophagic activity by 3-MA reversed the effects of SP on ROS and osteogenic levels. The present results indicated that autophagic activity participated in the regulation of differentiation fate of BMSCs and SP could promote osteogenic differentiation by activating autophagy, providing a more precise biological mechanism for its application in bone tissue engineering.
\end{abstract}

\section{Introduction}

The reconstruction of large-sized bone defects, caused by trauma, infection or tumor ablative surgery, remains a significant clinical challenge and the existing approaches exhibit

Correspondence to: $\mathrm{Dr}$ Rongcheng Mei, Department of Orthopaedics, Xiangyang Central Hospital, Affiliated Hospital of Hubei University of Arts and Science, 136 Jingzhou Street, Xiangyang, Hubei 441021, P.R. China

E-mail: meirch@163.com

*Contributed equally

Key words: autophagy, bone marrow stromal cells, substance P, osteogenesis, neuropeptide multiple drawbacks including donor site morbidity, lack of sufficient autografts, risk of disease transmission and immune rejection $(1,2)$. In the past few years a tremendous effort has been made to improve bone tissue engineering methods to overcome problems associated with existing grafts (1-3). It is well known that stem cells, especially mesenchymal stem cells (MSCs), have been used for bone tissue engineering for a considerable amount of time and bone mesenchymal stem cells (BMSCs) have been extensively investigated $(3,4)$. BMSCs can differentiate into adipocytes, chondrocytes and osteocytes in different microenvironments and the appropriate stimuli could enhance their ability of targeted differentiation $(5,6)$. However, the lineage commitment of BMSCs is a tightly regulated and well-orchestrated complex event, which has limited its clinical applications.

Multiple efforts have been devoted to modulate the microenvironment supporting BMSC osteogenic differentiation, including material surface functionalization by ECM analogues, drug or growth factor administration, and sustained release of bioactive molecules by gene modification, in which the well-controlled ROS generation has been revealed to play an important role (7-10). Substance P (SP), released predominantly by the peripheral terminal, is a conserved undecapeptide and a member of the tachykinin peptide family that acts as a sensory neurotransmitter and neuromodulator. Similar to growth factors, increasing studies have demonstrated that neuropeptides are critical for maintaining tissue homeostasis, including SP (11,12). Autophagy, an intracellular degradation and adaptive system, was confirmed to play important roles in regulating intercellular ROS levels and promoting neuropeptide function $(13,14)$. However, whether the interaction of SP and autophagy exists in regulating the differentiation fate of BMSCs remains elusive.

In the present study, the profile of ROS generation and autophagic activation was examined during BMSC osteogenic differentiation and their roles in SP-promoted BMSC osteogenic differentiation were further investigated. The results demonstrated that both ROS level and autophagic activity were increased with osteogenic induction. Furthermore, autophagic activity played an important role in restricting the excessive ROS generation mediating SP-enhanced BMSC osteogenic differentiation. 


\section{Materials and methods}

Isolation and culture of rat BMSCs. All animal experiments in this study were conducted in accordance with the Guidelines for Animal Experimentation and approved by the Ethics Committee of Xiangyang Central Hospital. BMSCs were harvested from femora and tibiae of Sprague-Dawley rats (six male, three months old, average weight $300 \mathrm{~g}$ ), purchased from the Laboratory Animal Center of Huazhong University of Science and Technology (Wuhan, China). The rats had ad libitum access to food and autoclaved water, and were housed at a constant temperature $\left(22-24^{\circ} \mathrm{C}\right)$, with $55 \%$ relative humidity, and a 12-h light/dark cycle. All efforts were made to minimize suffering and distress in this study. After being sacrificed by cervical dislocation (six male), the rats were soaked in $75 \%$ alcohol for $5 \mathrm{~min}$ and the femora and tibiae were separated and the connective tissue was removed. After cutting off both ends of the bones, the bone marrow was flushed out from medullary cavities by a syringe into complete culture media that consisted of Dulbecco's modified Eagle's medium with F12 nutrient mixture with $15 \%$ fetal bovine serum (FBS; both from Gibco; Thermo Fisher Scientific, Inc.) and $1 \%$ penicillin/streptomycin (Invitrogen; Thermo Fisher Scientific, Inc.). The bone marrow suspension was then plated in $25 \mathrm{~cm}$ culture bottles. The medium was changed every 2-3 days and cells were passaged when $80 \%$ confluent.

BMSC identification and osteogenic differentiation. After the second passage, the cells were collected and identified using fluorescently-labeled antibodies for BMSC markers: Allophycocyanin-conjugated CD29 (cat. no. 102225; BioLegend, Inc.); FITC-conjugated CD44 (cat. no. 203906; BioLegend, Inc.); FITC-conjugated CD45 (cat. no. 202205; BioLegend, Inc.); and phycoerythrin-conjugated CD34 (ab223930; Abcam). Briefly, the cells were incubated with corresponding antibodies for $30 \mathrm{~min}$ at $4^{\circ} \mathrm{C}$ in the dark and then washed with PBS. The expression levels of different cell surface markers were detected using FACSCalibur flow cytometer and analyzed using CellQuestPro version 5.1.1 software (BD Biosciences).

After detecting the purity, BMSCs from the second passage were used in subsequent experiments. To induce osteogenic differentiation, BMSCs were initially cultured in complete culture media as aforementioned. When the cells reached $80 \%$ confluence, osteogenic-inducing medium [ $\alpha$-MEM (Cyagen Biosciences) supplemented with $10 \% \mathrm{FBS}, 50 \mathrm{mg} / \mathrm{ml}$ ascorbate, $10 \mathrm{mM} \beta$-glycerophosphate, $100 \mathrm{nM}$ dexamethasone and $1 \%$ penicillin-streptomycin] was used. Lastly, the plates were stained with alizarin red and alkaline phosphatase at four time-points $(0,3,7$, and 14 days).

Treatment of rat BMSCs. Rat BMSCs were seeded on a 6-well culture plate. When the culture confluence reached $80 \%$, rat BMSCs were immediately incubated with osteogenic-inducing medium with or without SP $(10 \mathrm{nM})$, or pre-treated with 3-methyladenine (3-MA, $5 \mathrm{mM})$ and rapamycin (100 $\mathrm{nM})$ for $2 \mathrm{~h}$

Western blot analysis. The samples were lysed in cell lysis buffer for western blotting and IP (Beyotime Institute of
Biotechnology) supplemented with protease inhibitors (Beyotime Institute of Biotechnology), and the mixture was subjected to sonication at a low frequency $(20 \mathrm{kHz}, 50 \mathrm{~W}$, for $36 \mathrm{sec}$ on ice). After centrifugation (at 13,500 x g for $10 \min 4^{\circ} \mathrm{C}$ ), the supernatant was harvested and the protein concentration was assessed using a bicinchoninic acid protein assay kit (Beyotime Institute of Biotechnology). Equal amounts of samples by weight $(40 \mu \mathrm{g})$ from each sample were electrophoresed on 10-20\% SDS-PAGE and transferred to nitrocellulose membranes (both from Bio-Rad Laboratories, Inc.). After blocking in 5\% bovine serum albumin (Beyotime Institute of Biotechnology) for $2 \mathrm{~h}$ at room temperature, the membranes were incubated overnight at $4^{\circ} \mathrm{C}$ with primary antibodies for Runx2 (1:800; cat. no. ab76956; Abcam) and Osteocalcin (1:500; cat. no. ab13420; Abcam), LC3 (1:500; cat. no. 3868T; Cell Signaling Technology, Inc.), p62 (1:1,000; cat. no. 23214S; Cell Signaling Technology, Inc.), AMPK (1:800; cat. no. 2532S; Cell Signaling Technology, Inc.), p-AMPK (1:1,000; cat. no. 4184S; Cell Signaling Technology, Inc.), mTOR (1:800; cat. no. 2972S; Cell Signaling Technology, Inc.), p-mTOR (1:1,000; cat. no. 5536T; Cell Signaling Technology, Inc.) and GAPDH (1:1,000; cat. no. ab37168; Abcam). After rinsing with Tris-buffered saline supplemented with Tween, four times, the membranes were incubated with horseradish peroxidase-conjugated secondary antibodies goat anti-mouse and goat anti-rabbit immunoglobulin $\mathrm{G}(1: 2,000$; cat. no. SA00001-1 and cat. no. SA00001-2, respectively; ProteinTech Group, Inc.) for $1.5 \mathrm{~h}$ at room temperature. The protein bands were visualized using an ECL kit (Beyotime Institute of Biotechnology). Protein quantification was performed using Glyco Bandscan 5.0 software (ProZyme; Agilent Technologies, Inc.).

ROS assay. The intracellular total ROS levels were assessed by 2',7'-dichlorofluorescin diacetate (DCFH-DA; Beyotime Institute of Biotechnology) staining, which can be rapidly oxidized to a highly fluorescent compound. The samples were stained with $10 \mu \mathrm{M}$ DCFH-DA at $37^{\circ} \mathrm{C}$ for $20 \mathrm{~min}$, evaluated using FACSCalibur flow cytometer and analyzed using CellQuestPro version 5.1.1 software (BD Biosciences).

Alkaline phosphatase staining. To observe the osteogenic differentiation, the levels of alkaline phosphatase were examined by BCIP/NBT Alkaline Phosphatase Color Development Kit (Beyotime Institute of Biotechnology). The plates were washed twice with PBS and fixed at room temperature with 4\% paraformaldehyde for $5 \mathrm{~min}$. The plates were then washed three times with $\mathrm{ddH}_{2} \mathrm{O}$, stained at room temperature with BCIP/NBT working solution for $30 \mathrm{~min}$, washed three times with $\mathrm{ddH}_{2} \mathrm{O}$, and visualized using a fluorescence microscope (Olympus IX73; Olympus Corporation).

Alizarin Red staining. The mineralization of osteogenic induction was detected by Alizarin red staining. The plates were washed twice with PBS and fixed at room temperature with $4 \%$ formaldehyde for $10 \mathrm{~min}$. The plates were then washed gently three times with $\mathrm{ddH}_{2} \mathrm{O}$, stained at room temperature with 1\% Alizarin Red (Sigma Aldrich) for $10 \mathrm{~min}$, and visualized using a fluorescence microscope (Olympus IX73; Olympus Corporation). 
A

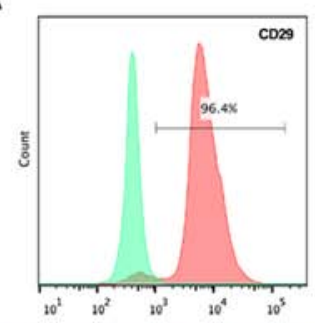

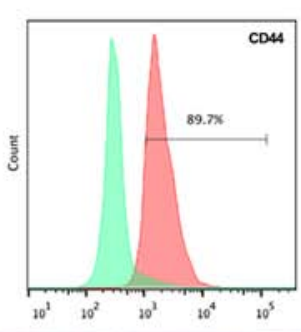

B
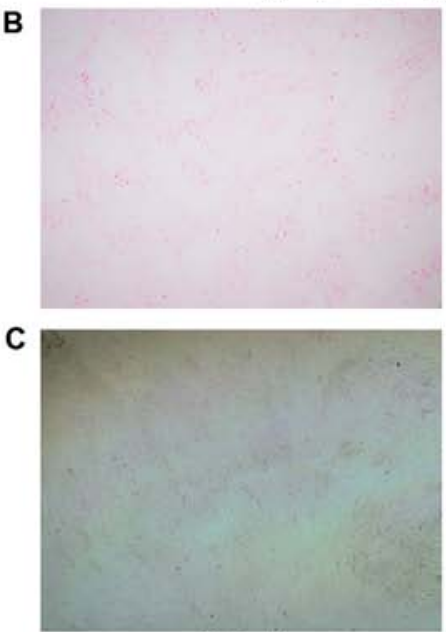

OI day 0
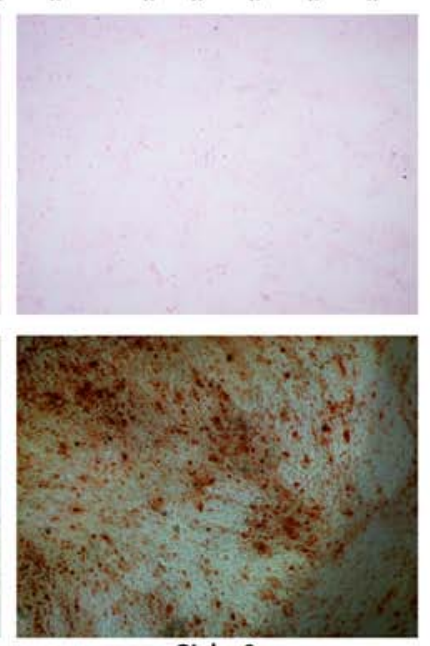

OI day 3
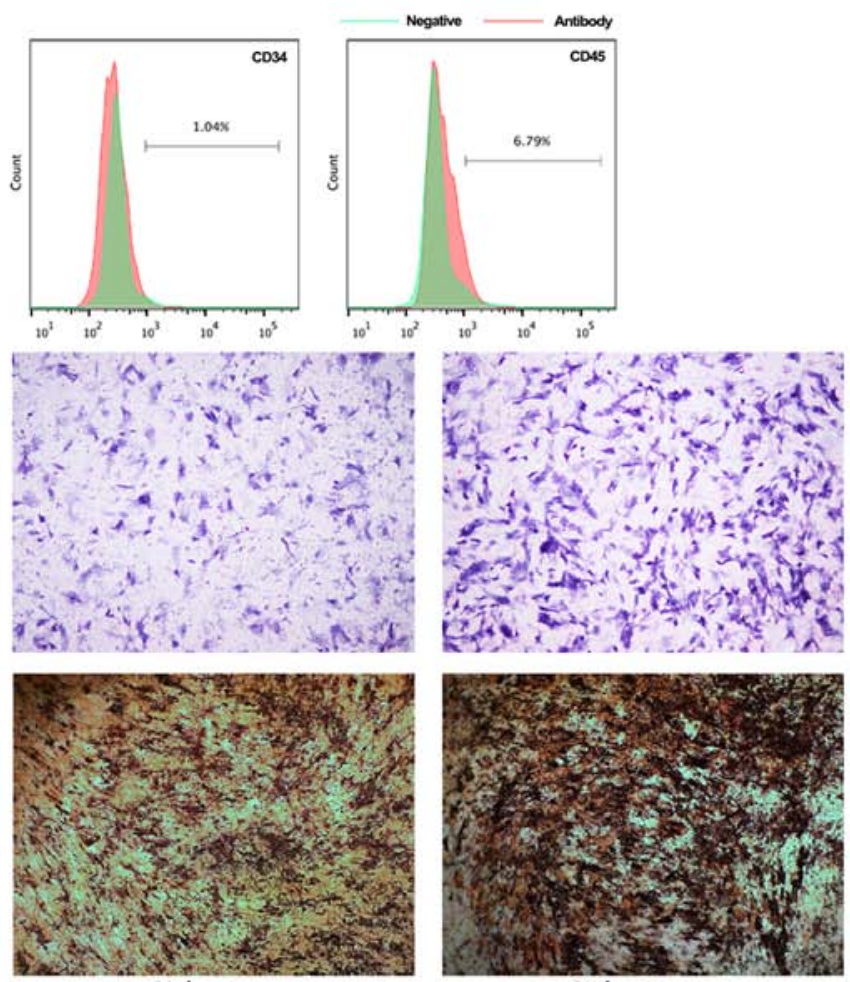

Ol day 7

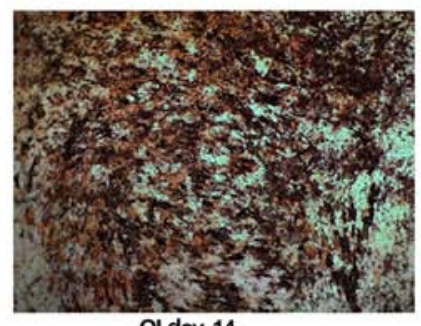

Ol day 14

Figure 1. Identification and characterization of rat BMSCs. (A) Flow cytometric results revealed high expression of CD29 (96.4\%) and CD44 (89.7\%) and negligible expression of CD34 (1.04\%) and CD45 (6.79\%). (B) Representative images of ALP staining, evaluated by NBT-formazan formation (magnification, $\mathrm{x} 40$ ). (C) Representative images of Alizarin Red staining, evaluated by mineralized nodules (magnification, x40). BMSCs, bone mesenchymal stem cells; ALP, alkaline phosphatase.

Statistical analysis. Data were analyzed using SPSS software, version 17.0 (SPSS, Inc.) and are reported as the mean \pm standard deviation of at least three independent experiments. The differences between two groups were analyzed using a Student's t-test and comparisons among multiple groups were performed using one-way analysis of variance followed by Tukey's post hoc test. $\mathrm{P}<0.05$ was considered to indicate a statistically significant difference.

\section{Results}

Identification and characterization of rat BMSCs. The BMSCs at second passage, were a homogenous population and exhibited a spindle-shaped morphology. Furthermore, BMSC phenotypes were identified using flow cytometric analysis. The cells were positive for typical BMSC markers CD29 (96.4\%) and CD44 (89.7\%), with concomitant absence of CD34 (1.04\%) and CD45 (6.79\%) (Fig. 1A). In addition, alkaline phosphatase (ALP) staining and Alizarin red staining were performed to detect osteogenic differentiation after culture with osteogenic-inducing medium. As revealed in Fig. 1B, the NBT-formazan formation with BCIP/NBT working solution staining was significantly increased at 7 and 14 days, indicating the ALP activity in BMSCs. Similarly, the Alizarin Red staining results revealed that increased mineralized nodules were formed along with the prolongation of osteogenic-inducing term (Fig. 1C). Collectively, these data revealed the purity and osteogenic ability of rat BMSCs isolated in our present experiments.
Autophagy has a potential role in reducing ROS production and promoting osteogenic differentiation. While excessive ROS is deleterious to cell function, moderate levels of ROS have been reported to participate in regulating BMSC differentiation fate (15). It is well known that autophagy plays an important role in maintaining cellular homeostasis, including ROS generation. In the present study, we examined the roles of autophagy and ROS in regulating BMSC differentiation fate. The ROS levels were first detected during osteogenic differentiation. As anticipated, flow cytometric results revealed that osteogenic-induction significantly increased the ROS level in BMSCs (Fig. 2A). Next, whether osteogenic induction could induce autophagy in BMSCs was examined. LC3 II/LC3 I conversion is a well-known biomarker for indicating the formation of autophagosomes and p62 for the degradation of autolysosomes. As presented in Fig. 2B, the western blotting results revealed that osteogenic induction significantly promoted the LC3 II conversion from LC3 I and decreased the p62 levels, which indicated increased autophagic activity.

To further examine whether autophagy participates in regulating ROS production and the differentiation fate of BMSCs during osteogenic-induction, BMSCs were pre-treated with autophagy inhibitor 3-MA or activator rapamycin for $2 \mathrm{~h}$. The results revealed that pre-treatment with 3-MA prior to osteogenic induction significantly increased ROS production in BMSCs (Fig. 2C) and decreased the protein levels of Runx2 and osteocalcin, which are markers of osteogenesis (Fig. 2D). Conversely, pre-treatment with autophagy activator rapamycin attenuated ROS production in BMSCs during 
A
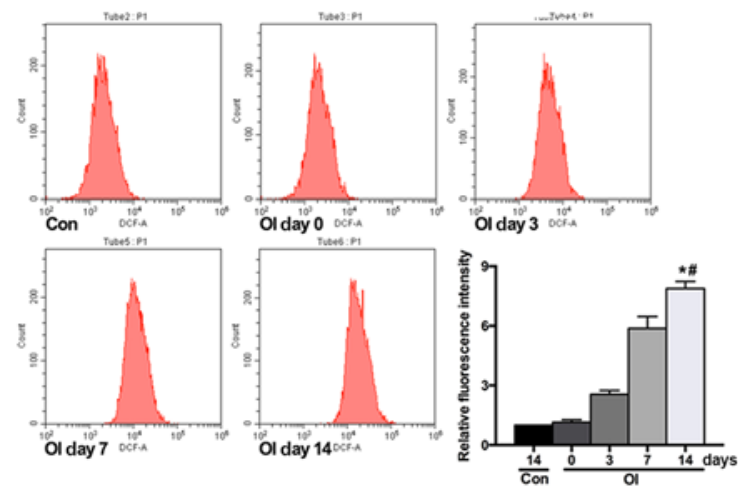

B
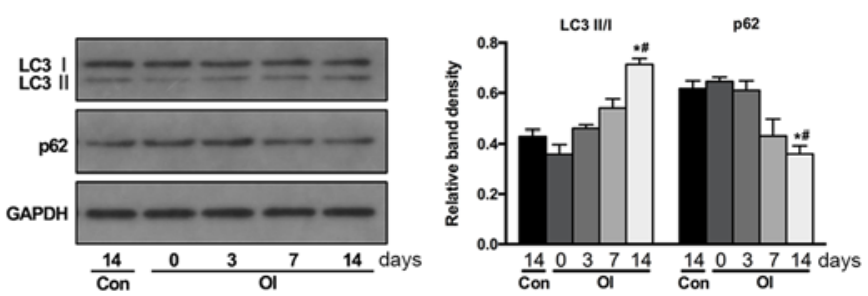

D
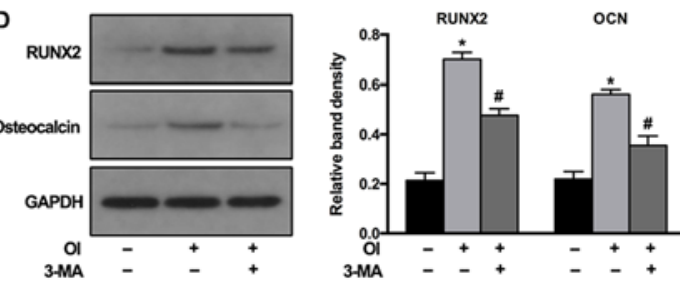

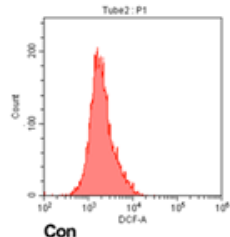

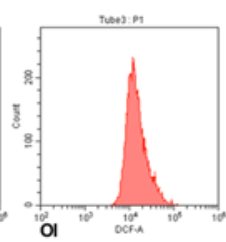

$\mathrm{Ol+3 \textrm {MA }}$
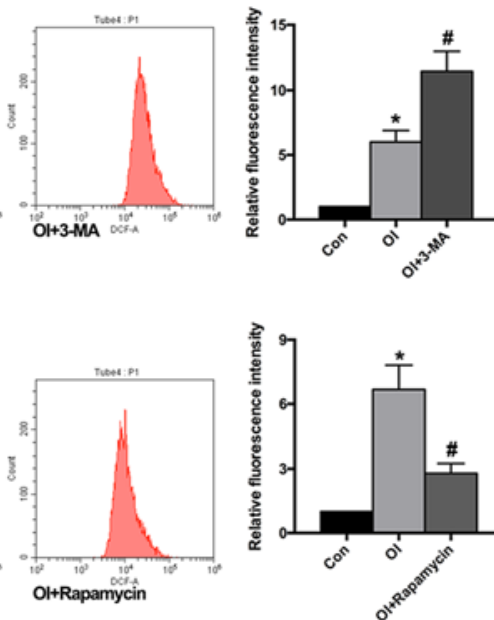

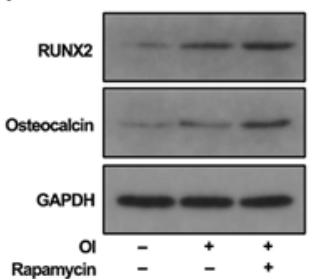

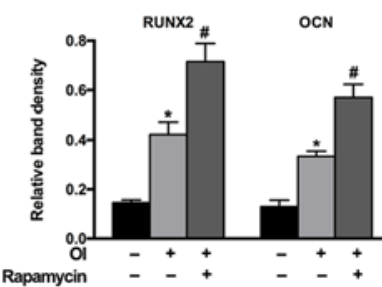

Figure 2. Increased ROS generation is attenuated by autophagic activity. (A) Cellular ROS was stained by DCFH-DA and the representative peak charts of flow cytometry and quantitative analysis are shown. ${ }^{*} \mathrm{P}<0.05$ vs. day $0 ;{ }^{*} \mathrm{P}<0.05$ vs. Con. (B) Representative western blotting assay and quantitation of the LC3II/LC3I ratio and p62 protein level. "P<0.05 vs. day $0 ;{ }^{\#} \mathrm{P}<0.05$ vs. Con. (C and D) Autophagic inhibition by $3-\mathrm{MA}$ increased ROS generation and decreased the levels of Runx 2 and osteocalcin. ${ }^{*} \mathrm{P}<0.05$ vs. Con; ${ }^{\#} \mathrm{P}<0.05$ vs. OI. (E and F) Autophagic activation by rapamycin attenuated ROS generation and increased the levels of Runx 2 and osteocalcin. ${ }^{*} \mathrm{P}<0.05$ vs. Con; ${ }^{\prime} \mathrm{P}<0.05$ vs. OI. ROS, reactive oxygen species; Con, control group; 3 -MA, 3 -methyladenine; OI, osteogenic induction group.

osteogenic-induction (Fig. 2E) and further increased the protein levels of Runx 2 and osteocalcin (Fig. 2F). These results demonstrated that autophagic activity could control the ROS level in BMSCs during osteogenic induction and promote their osteogenic differentiation.

Substance $P$ administration regulates $R O S$ generation in BMSCs via autophagic activation. The neuropeptide SP has been demonstrated to have a potentially protective role in multiple tissue disorders, partially by regulating inflammatory response or ROS generation (16-18). In the present study, the role of autophagy in these processes was further investigated. As revealed in Fig. 3A and B, SP treatment significantly increased the ratio of $\mathrm{p}-\mathrm{AMPK} / \mathrm{AMPK}$ and decreased the ratio of $\mathrm{p}-\mathrm{mTOR} / \mathrm{mTOR}$, which are classic mediators of autophagy activity (19). In addition, the LC3 II/LC3 I ratio was also enhanced by SP treatment, along with p62 degradation (Fig. 3C). These results demonstrated that SP could induce autophagy by promoting AMPK and suppressing mTOR activation. To further investigate the role of SP and autophagy interaction in ROS production during osteogenic induction, BMSCs were pre-treated with (3-MA). As a result, the autophagic activation induced by SP treatment was effectively inhibited by 3-MA (Fig. 3C). Subsequently, it was observed that SP treatment had a suppressive effect on ROS generation during BMSC osteogenic induction, which could be attenuated along with autophagic inhibition (Fig. 3D). Collectively, these findings indicated that SP administration could regulate ROS generation during BMSC differentiation by inducing autophagic activity.

Substance $P$ administration promotes BMSC osteogenic differentiation via autophagic activation. The effects of SP administration on BMSC differentiation and the role of autophagy were further examined. As revealed in Fig. 4A, the addition of SP with osteogenic-inducing medium significantly increased the protein levels of Runx 2 and osteocalcin, which were attenuated by 3-MA pre-treatment. The results of ALP and Alizarin red staining also confirmed that the enhanced osteogenic differentiation by the addition of SP was decreased by 3-MA pre-treatment, visualized by reduced formation of NBT-formazan and mineralized nodules (Fig. 4B).

\section{Discussion}

Bone tissue engineering and therapeutic strategies, developed at the pre-clinical and clinical levels are largely limited to treat small size defects based on novel functional materials and are not capable of treating large-size and compromised defects due to innate limitations in bone inductive properties of the 

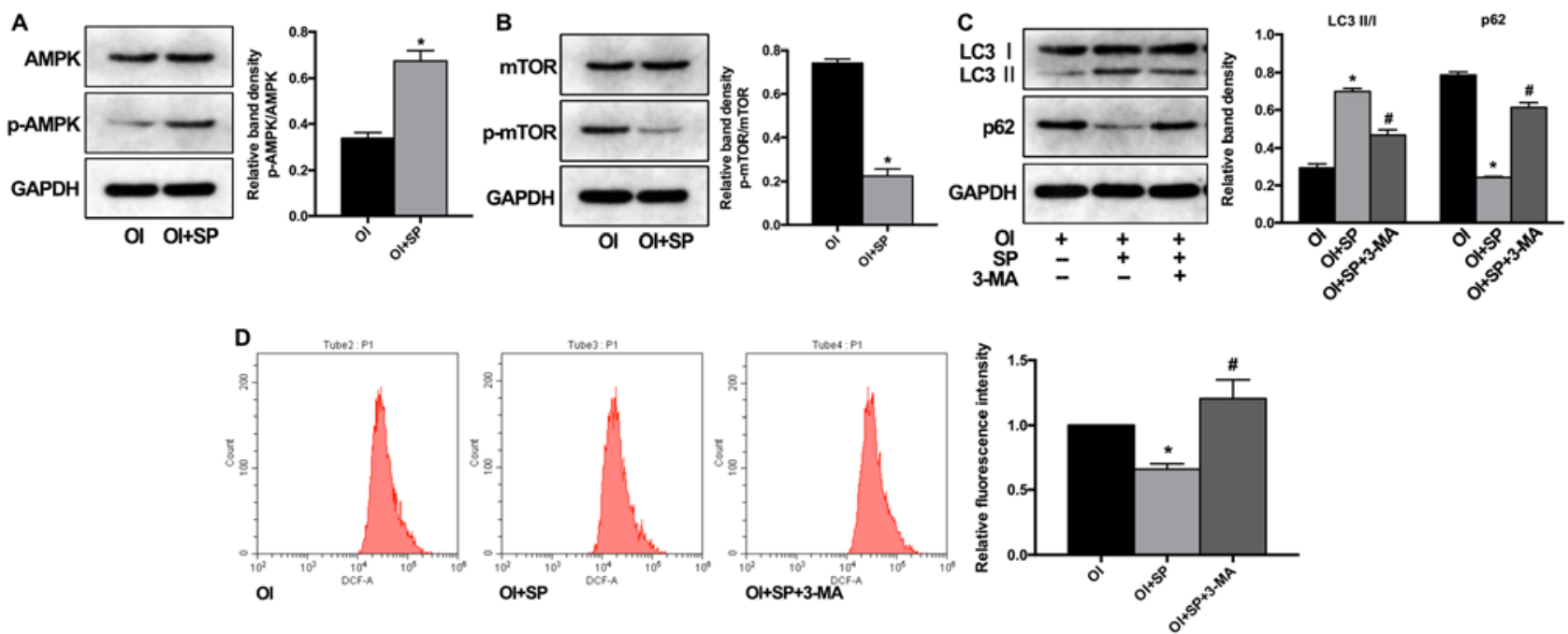

Figure 3. SP treatment enhances the autophagic activity and decreases the ROS levels. (A and B) The co-culture of SP with osteogenic-inducing medium increased the ratio of $\mathrm{p}$-AMPK/AMPK and decreased the ratio of $\mathrm{p}-\mathrm{mTOR} / \mathrm{mTOR}$. " $\mathrm{P}<0.05$ vs. OI. (C) SP-induced LC3II/LC3I conversion and p62 degradation were inhibited by 3 -MA pre-treatment. ${ }^{*} \mathrm{P}<0.05$ vs. OI; ${ }^{~} \mathrm{P}<0.05$ vs. OI+SP. (D) SP-suppressed ROS generation was inhibited by 3-MA pre-treatment. ${ }^{\text {*P}} \mathrm{P}<0.05$ vs. OI; ${ }^{\text {P }}<0.05$ vs. OI + SP. SP, substance P; ROS, reactive oxygen species; OI, osteogenic induction group; 3-MA, 3-methyladenine.
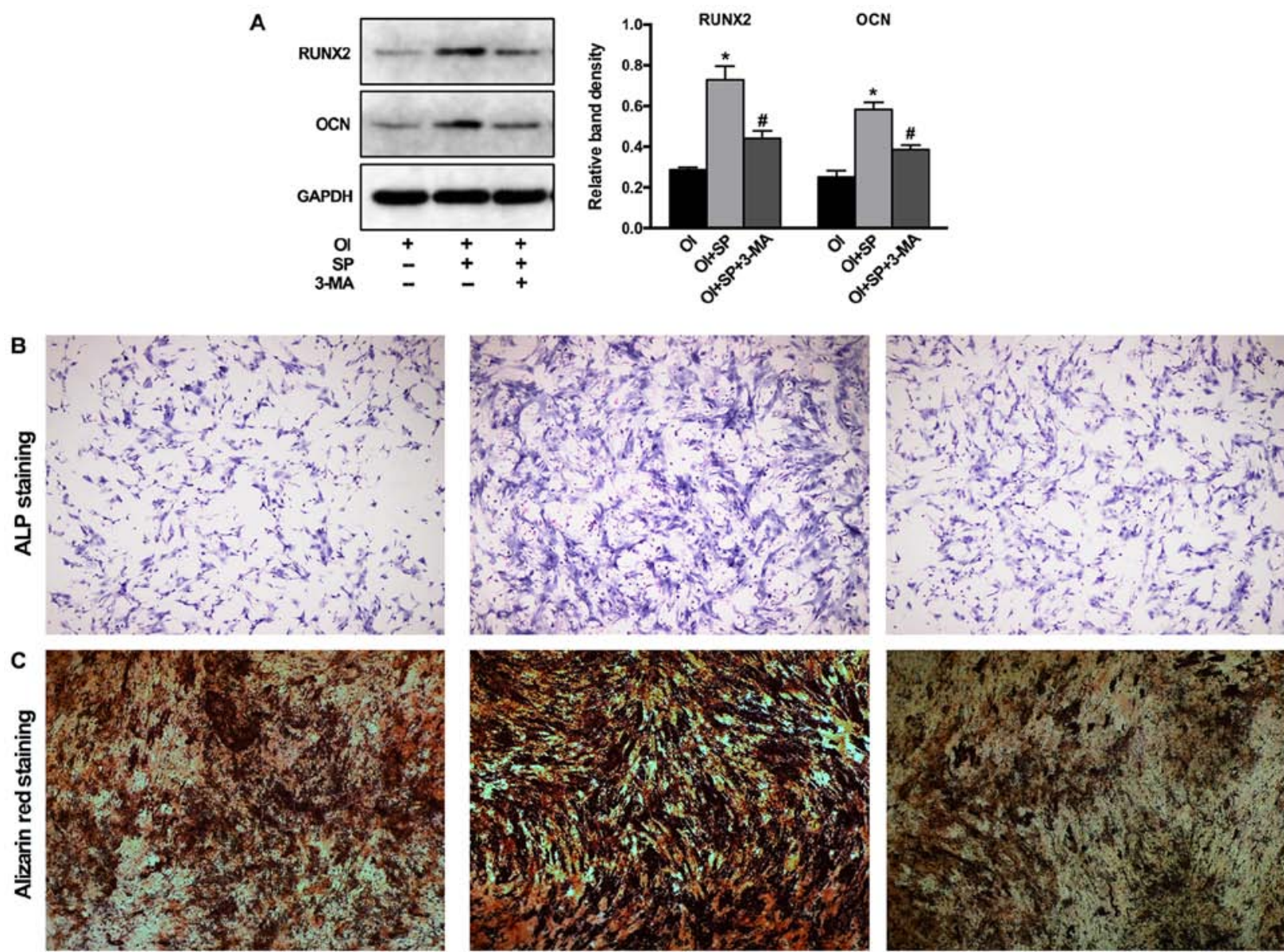

OI

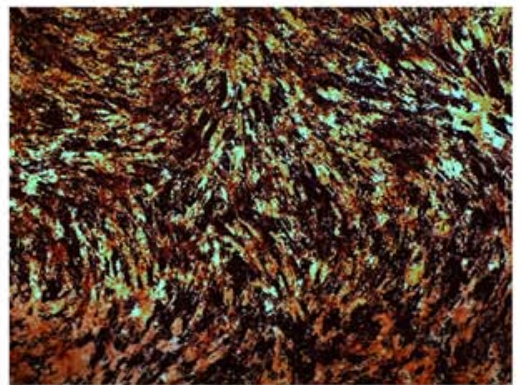

$\mathrm{Ol}+\mathrm{SP}$

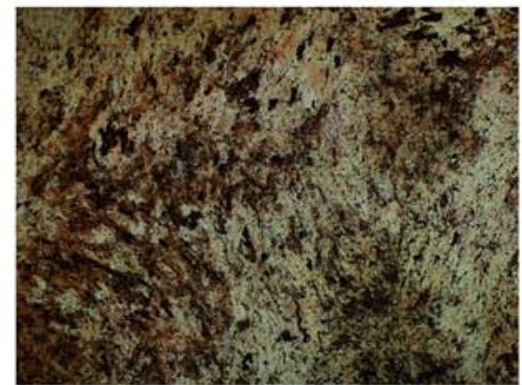

$\mathrm{Ol}+\mathrm{SP}+3-\mathrm{MA}$

Figure 4. SP treatment promotes BMSC osteogenic differentiation via activation of autophagy. (A) The upregulated Runx2 and osteocalcin levels by SP treatment were inhibited by 3 -MA pre-treatment. ${ }^{~} \mathrm{P}<0.05$ vs. OI. ${ }^{\#} \mathrm{P}<0.05$ vs. OI $+\mathrm{SP}$. (B and C) SP treatment promoted the osteogenic differentiation, visualized as increased NBT-formazan and mineralized nodule formation and autophagic inhibition by 3-MA ameliorated this response (magnification, $\mathrm{x} 40$ ). SP, substance P; BMSC, bone mesenchymal stem cell; 3-MA, 3-methyladenine; OI, osteogenic induction group.

materials (20). In view of this, biomolecule-based and cell-based strategies are being continuously evaluated (3) but are also particularly difficult to translate to clinical applications due to complex mechanisms involved. The present study demonstrated 
that autophagy played an important role in restricting the excessive ROS generation and promoting BMSCs osteogenic differentiation. Furthermore, it was revealed that the administration of neuropeptide SP could enhance autophagic activity and subsequently promote BMSC osteogenic differentiation.

Endogenous reactive oxygen species (ROS) are produced as by-products of the mitochondrial electron transport chain, and can be greatly elevated with increase in mitochondrial respiration that is essential to meet the energy demand of differentiating cells. ROS generation is however not just a consequence of differentiation but plays a critical role in governing the fate of stem cell differentiation $(21,22)$. Elevated ROS in BMSCs has been reported to promote adipogenesis, and scavenging of the ROS restores the osteogenic capacity (23). Notably in the present study, an apparent increase of ROS levels was observed during osteogenic-induction, which was an outcome contradictory to the results reported in a previous study (24). It is considered that this difference may mainly result from the addition of dexamethasone to the osteogenesis-inducing medium $(25,26)$. This osteogenesis-inducing medium that could increase ROS levels, mimicked most oxidative stress microenvironments that are associated with bone-defect diseases $(15,27)$, which may provide a closer insight for investigating the regulatory mechanisms of BMSC differentiation. Similarly, it has been reported that the non-direct crosstalk between acute myeloid leukemia cells and BMSCs mediated by the local cytokine network could alter the global gene expression profile of BMSCs (28), which indicated that the disease-specific microenvironments are worthy of more attention when tissue engineering products are developed and used for reconstructing bone defects.

Autophagy is a critical intracellular degradation system for maintaining cellular homeostasis. It has been reported that increased ROS levels stimulated autophagy that acted as a feedback control for excessive ROS production (29). Similarly, in the present study, a concomitant induction of autophagic activity was observed with increased ROS levels and pharmacological activation/inhibition of autophagy could attenuate/aggravate ROS generation. Furthermore, we observed that autophagic activity was involved in the regulation of BMSC osteogenic differentiation. Considering the importance of redox status in stem cell differentiation, multiple studies have emphasized the crucial role of well-orchestrated coordination of cellular antioxidant systems $(22,30,31)$, which are not always in a favorable environment $(32,33)$. In the present study, it was observed that autophagy functioned well as a candidate for maintaining redox homeostasis.

Growth factors, in increasing numbers, have forayed into pre-clinical trials or clinical applications as a result of extensive investigations (3). Neuropeptides are also critical for maintaining tissue homeostasis and SP has been demonstrated to have an osteogenic effect on BMSCs $(11,12,34)$, whereas few studies have reported their clinical trials or application due to the unclear working mechanisms. Previous studies have demonstrated that autophagic activity was involved in mediating the biological function of neuropeptides $(13,14)$. However, its role in SP function and their interaction in BMSC differentiation has remained unclear. AMPK and mTOR are two classic pathways that elicit a coordinated response to stimuli and regulation of autophagic activity (19). In our present study, it was revealed that SP administration increased the protein ratio of $\mathrm{p}$-AMPK/AMPK while reducing the protein ratio of $\mathrm{p}-\mathrm{mTOR} / \mathrm{mTOR}$ and activated autophagy in BMSCs. Suppression of ROS generation resulted in increased osteogenic differentiation. Furthermore, pharmacological inhibition of autophagy ameliorated the osteogenic effect of SP, indicating that SP could orchestrate autophagic activity and thereby promote BMSC osteogenic differentiation.

However, the limitations that involved the pharmacological modulation of autophagic activity cannot be neglected. Multiple chemical agents that are currently available to activate or inhibit autophagy have limited specificity for the autophagic process, including 3-MA and rapamycin (35). Addressing these complex effects in cell- and disease-specific models is key to developing clinical strategies using autophagic modulators. Although the present study revealed the autophagy-activated and osteogenic effects of SP administration, more potential molecules that interact with SP and the involved mechanisms require further in vivo investigations in the future.

In summary, a well-regulated redox status is involved in determining BMSC differentiation fate and autophagic activity is essential for maintaining redox homeostasis. Additionally, it was observed that SP administration promotes autophagic activity by regulating the AMPK and mTOR pathways and further enhances the osteogenic differentiation of BMSCs. Thus, we not only propose the application of SP in bone tissue engineering and other therapeutic strategies for bone-damaging diseases, but also postulate a potential molecular mechanism by which SP imparts its beneficial effects.

\section{Acknowledgements}

Not applicable.

\section{Funding}

The present study was supported by the Scientific Action Plans for the Prevention and Treatment of Major Diseases National Health Commission of the People's Republic of China-Trauma Repair (ZX-01-C2016156).

\section{Availability of data and materials}

All data used in the present study are included in this published article.

\section{Authors' contributions}

WG and HMS designed the study protocol, conducted experiments and wrote the first manuscript. XMZ, WT and YC helped to design the study, and collected and analysed data. RCM, contributed to study design, gave final approval of study protocol, and extensively reviewed and revised manuscript. All authors read and approved the manuscript and agree to be accountable for all aspects of the research in ensuring that the accuracy or integrity of any part of the work are appropriately investigated and resolved.

\section{Ethics approval and consent to participate}

The animal experiments were performed following a protocol approved by the Animal Experimentation Committee of 
Xiangyang Central Hospital, Affiliated Hospital of Hubei University of Arts and Science.

\section{Patient consent for publication}

Not applicable.

\section{Competing interests}

The authors declare that they have no competing interests.

\section{References}

1. Petite H, Viateau V, Bensaïd W, Meunier A, de Pollak C, Bourguignon M, Oudina K, Sedel L and Guillemin G: Tissue-engineered bone regeneration. Nat Biotechnol 18: 959-963, 2000.

2. Pobloth AM, Checa S, Razi H, Petersen A, Weaver JC, Schmidt-Bleek K, Windolf M, Tatai AÁ, Roth CP, Schaser KD et al: Mechanobiologically optimized 3D titanium-mesh scaffolds enhance bone regeneration in critical segmental defects in sheep. Sci Transl Med 10: pii: eaam8828, 2018.

3. Ho-Shui-Ling A, Bolander J, Rustom LE, Johnson AW, Luyten FP and Picart C: Bone regeneration strategies: Engineered scaffolds, bioactive molecules and stem cells current stage and future perspectives. Biomaterials 180: 143-162, 2018.

4. Marolt D, Knezevic M and Novakovic GV: Bone tissue engineering with human stem cells. Stem Cell Res Ther 1: 10, 2010.

5. Man Z, Yin L, Shao Z, Zhang X, Hu X, Zhu J, Dai L, Huang H, Yuan L,Zhou C, et al: The effects of co-delivery of BMSC-affinity peptide and rhTGF-beta1 from coaxial electrospun scaffolds on chondrogenic differentiation. Biomaterials 35: 5250-5260, 2014.

6. Cai S, Tsui YP, Tam KW, Shea GK, Chang RS, Ao Q, Shum DK and Chan YS: Directed differentiation of human bone marrow stromal cells to Fate-committed schwann cells. Stem Cell Reports 9: 1097-1108, 2017.

7. Chen W, Shen X, Hu Y, Xu K, Ran Q, Yu Y, Dai L, Yuan Z, Huang L, Shen T and Cai K: Surface functionalization of titanium implants with chitosan-catechol conjugate for suppression of ROS-induced cells damage and improvement of osteogenesis. Biomaterials 114: 82-96, 2017.

8. Liu Z, Yuan X, Fernandes G, Dziak R, Ionita CN, Li C, Wang C and Yang S: The combination of nano-calcium sulfate/platelet rich plasma gel scaffold with BMP2 gene-modified mesenchymal stem cells promotes bone regeneration in rat critical-sized calvarial defects. Stem Cell Res Ther 8: 122, 2017.

9. Li J, Zhang J, Chen Y, Kawazoe N and Chen G: TEMPOconjugated gold nanoparticles for reactive oxygen species scavenging and regulation of stem cell differentiation. ACS Appl Mater Interfaces 9: 35683-35692, 2017.

10. Kumar Y,Biswas T, Thacker G, Kanaujiya JK, Kumar S, Shukla A, Khan K, Sanyal S, Chattopadhyay N, Bandyopadhyay A and Trivedi AK: BMP signaling-driven osteogenesis is critically dependent on Prdx-1 expression-mediated maintenance of chondrocyte prehypetrophy. Free Radic Biol Med 118: 1-12, 2018.

11. Dioufa N, Schally AV, Chatzistamou I, Moustou E, Block NL, Owens GK, Papavassiliou AG and Kiaris H: Acceleration of wound healing by growth hormone-releasing hormone and its agonists. Proc Natl Acad Sci USA 107: 18611-18615, 2010.

12. Lim JE, Chung E and Son Y: A neuropeptide, Substance-P, directly induces tissue-repairing M2 like macrophages by activating the PI3K/Akt/mTOR pathway even in the presence of IFN $\gamma$. Sci Rep 7: 9417, 2017.

13. Oh TS, Cho H, Cho JH, Yu SW and Kim EK: Hypothalamic AMPK-induced autophagy increases food intake by regulating NPY and POMC expression. Autophagy 12: 2009-2025, 2016.

14. Aveleira CA, Botelho M, Carmo-Silva S, Pascoal JF, FerreiraMarques M, Nóbrega C, Cortes L, Valero J, Sousa-Ferreira L, Álvaro AR, et al: Neuropeptide Y stimulates autophagy in hypothalamic neurons. Proc Natl Acad Sci USA 112: E1642-E1651, 2015.

15. Atashi F, Modarressi A and Pepper MS: The role of reactive oxygen species in mesenchymal stem cell adipogenic and osteogenic differentiation: A review. Stem Cells Dev 24: 1150-1163, 2015.

16. Hong HS and Son Y: Substance P ameliorates collagen II-induced arthritis in mice via suppression of the inflammatory response. Biochem Biophys Res Commun 453: 179-184, 2014.
17. Kim SJ, Kim JE, Kim SH, Kim SJ, Jeon SJ, Kim SH and Jung Y: Therapeutic effects of neuropeptide substance P coupled with self-assembled peptide nanofibers on the progression of osteoarthritis in a rat model. Biomaterials 74: 119-130, 2016.

18. Yang L, Di G, Qi X, Qu M, Wang Y, Duan H, Danielson P, Xie L and Zhou Q: Substance P promotes diabetic corneal epithelial wound healing through molecular mechanisms mediated via the neurokinin-1 receptor. Diabetes 63: 4262-4274, 2014.

19. Klionsky DJ, Abdelmohsen K, Abe A, Abedin MJ, Abeliovich H, Acevedo Arozena A, Adachi H, Adams CM, Adams PD, Adeli $\mathrm{K}$, et al: Guidelines for the use and interpretation of assays for monitoring autophagy 3rd edition). Autophagy 12: 1-222, 2016.

20. Roffi A, Krishnakumar GS, Gostynska N, Kon E, Candrian C and Filardo G: The role of Three-dimensional scaffolds in treating long bone defects: Evidence from preclinical and clinical Literature-A systematic review. Biomed Res Int 2017: 8074178, 2017.

21. Tormos KV, Anso E, Hamanaka RB, Eisenbart J, Joseph J, Kalyanaraman B and Chandel NS: Mitochondrial complex III ROS regulate adipocyte differentiation. Cell Metab 14: 537-544, 2011.

22. Wang $K$, Zhang $T$, Dong $Q$, Nice EC, Huang $C$ and Wei $Y$ : Redox homeostasis: The linchpin in stem cell self-renewal and differentiation. Cell Death Dis 4: e537, 2013.

23. Chen H, Liu X, Chen H, Cao J, Zhang L, Hu X and Wang J: Role of SIRT1 and AMPK in mesenchymal stem cells differentiation. Ageing Res Rev 13: 55-64, 2014.

24. Chen CT, Shih YR, Kuo TK, Lee OK and Wei YH: Coordinated changes of mitochondrial biogenesis and antioxidant enzymes during osteogenic differentiation of human mesenchymal stem cells. Stem Cells 26: 960-968, 2008.

25. Zhang S, Liu Y and Liang Q: Low-dose dexamethasone affects osteoblast viability by inducing autophagy via intracellular ROS. Mol Med Rep 17: 4307-4316, 2018.

26. Shen C, Cai GQ, Peng JP and Chen XD: Autophagy protects chondrocytes from glucocorticoids-induced apoptosis via ROS/Akt/FOXO3 signaling. Osteoarthritis Cartilage 23: 2279-2287, 2015.

27. Wang L, Zhao X, Wei BY, Liu Y, Ma XY, Wang J, Cao PC, Zhang Y, Yan YB, Lei $\mathrm{W}$ and Feng YF: Insulin improves osteogenesis of titanium implants under diabetic conditions by inhibiting reactive oxygen species overproduction via the PI3K-Akt pathway. Biochimie 108: 85-93, 2015.

28. Reikvam H,Brenner AK, Hagen KM,Liseth K, Skrede S, Hatfield KJ and Bruserud $\varnothing$ : The cytokine-mediated crosstalk between primary human acute myeloid cells and mesenchymal stem cells alters the local cytokine network and the global gene expression profile of the mesenchymal cells. Stem Cell Res 15: 530-541, 2015.

29. Gómez-Puerto MC, Verhagen LP, Braat AK, Lam EW, Coffer PJ and Lorenowicz MJ: Activation of autophagy by FOXO3 regulates redox homeostasis during osteogenic differentiation. Autophagy 12: 1804-1816, 2016.

30. Kim H, Lee YD, Kim HJ, Lee ZH and Kim HH: SOD2 and Sirt3 control osteoclastogenesis by regulating mitochondrial ROS. J Bone Miner Res 32: 397-406, 2017.

31. Wang Y, Chen G, Yan J, Chen X, He F, Zhu C, Zhang J, Lin J, Pan G, Yu J, et al: Upregulation of SIRT1 by kartogenin enhances antioxidant functions and promotes osteogenesis in human mesenchymal stem cells. Oxid Med Cell Longev 2018: $1368142,2018$.

32. Liao L, Su X, Yang X, Hu C, Li B, Lv Y, Shuai Y, Jing H, Deng Z and Jin Y: TNF- $\alpha$ inhibits FoxO1 by upregulating miR-705 to aggravate oxidative damage in bone marrow-derived mesenchymal stem cells during osteoporosis. Stem Cells 34: 1054-1067, 2016.

33. Abnosi MH and Yari S: The toxic effect of gallic acid on biochemical factors, viability and proliferation of rat bone marrow mesenchymal stem cells was compensated by boric acid. J Trace Elem Med Biol 48: 246-253, 2018.

34. Fu S, Mei G, Wang Z, Zou ZL, Liu S, Pei GX, Bi L and Jin D: Neuropeptide substance P improves osteoblastic and angiogenic differentiation capacity of bone marrow stem cells in vitro. Biomed Res Int 2014: 596023, 2014.

35. Galluzzi L, Bravo-San Pedro JM, Levine B, Green DR and Kroemer G: Pharmaological modulation of autophagy: Therapeutic potential and persisting obstacles. Nat Rev Drug Discov 16: 487-511, 2017.

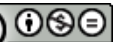

This work is licensed under a Creative Commons Attribution-NonCommercial-NoDerivatives 4.0 International (CC BY-NC-ND 4.0) License. 